\title{
Analytical Assessment of Simultaneous Parallel Approach Feasibility from Total System Error
}

\author{
Michael M. Madden * \\ NASA Langley Research Center, Hampton, VA, 23681
}

\begin{abstract}
In a simultaneous paired approach to closely-spaced parallel runways, a pair of aircraft flies in close proximity on parallel approach paths. The aircraft pair must maintain a longitudinal separation within a range that avoids wake encounters and, if one of the aircraft blunders, avoids collision. Wake avoidance defines the rear gate of the longitudinal separation. The lead aircraft generates a wake vortex that, with the aid of crosswinds, can travel laterally onto the path of the trail aircraft. As runway separation decreases, the wake has less distance to traverse to reach the path of the trail aircraft. The total system error of each aircraft further reduces this distance. The total system error is often modeled as a probability distribution function. Therefore, Monte-Carlo simulations are a favored tool for assessing a "safe" rear-gate. However, safety for paired approaches typically requires that a catastrophic wake encounter be a rare one-in-a-billion event during normal operation. Using a Monte-Carlo simulation to assert this event rarity with confidence requires a massive number of runs. Such large runs do not lend themselves to rapid turn-around during the early stages of investigation when the goal is to eliminate the infeasible regions of the solution space and to perform trades among the independent variables in the operational concept. One can employ statistical analysis using simplified models more efficiently to narrow the solution space and identify promising trades for more in-depth investigation using MonteCarlo simulations. These simple, analytical models not only have to address the uncertainty of the total system error but also the uncertainty in navigation sources used to alert an abort of the procedure. This paper presents a method for integrating total system error, procedure abort rates, avionics failures, and surveillance errors into a statistical analysis that identifies the likely feasible runway separations for simultaneous paired approaches.
\end{abstract}

\section{Nomenclature}

$\sigma \quad=$ standard deviation

$\sigma_{\Delta \mathrm{V}} \quad=$ standard deviation of the airspeed difference between paired aircraft

$\sigma_{\Delta \mathrm{x}} \quad=$ standard deviation of the longitudinal separation due to deviation in airspeed difference

$\sigma_{\mathrm{ALE}}=$ standard deviation in ADS-B position uncertainty due to latency

$\sigma_{\mathrm{EPU}}=$ standard deviation of the estimated position uncertainty for ADS-B

$\sigma_{\mathrm{FTE}} \quad=$ standard deviation of the flight technical error along an axis (lateral or longitudinal)

$\sigma_{\mathrm{NE}} \quad=$ standard deviation of the navigation error along an axis (lateral or longitudinal)

$\sigma_{\mathrm{obs}} \quad=$ standard deviation in the observed longitudinal separation

$\sigma_{\text {sep }} \quad=$ total standard deviation in longitudinal separation performance

$\Delta \mathrm{Y} \quad=$ the true lateral distance between aircraft

$\Delta \mathrm{Y}_{\text {runway }}=$ minimum feasible runway separation

$\mathrm{ADS}-\mathrm{B}=$ Automatic Dependent Surveillance - Broadcast

AGL $\quad=$ above ground level

$\mathrm{ALE}=\mathrm{ADS}-\mathrm{B}$ latency error

* Chief Scientist, Simulation Development and Analysis Branch, Mail Stop 125B, Senior Member AIAA. 


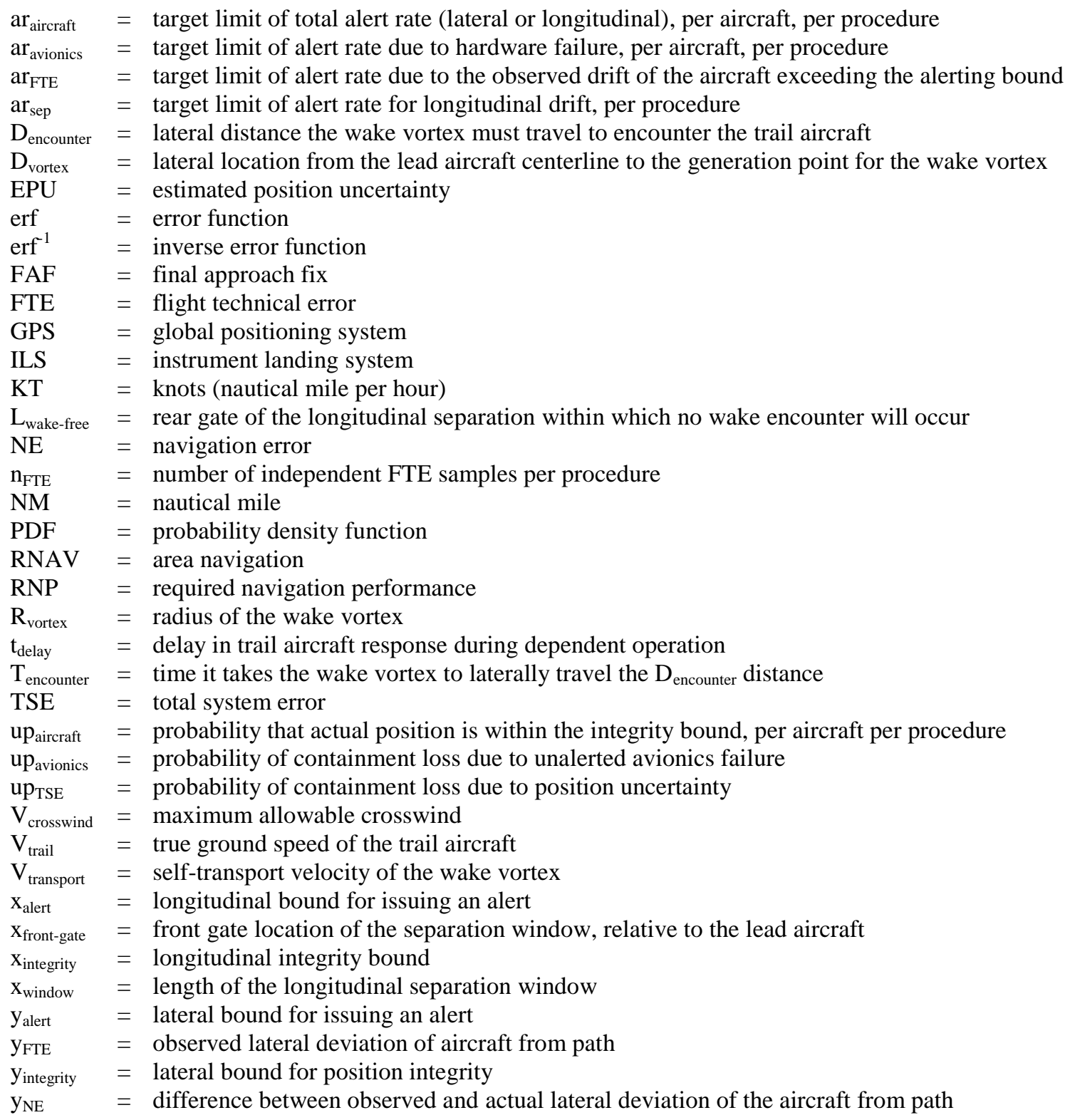

\section{Introduction}

THE Closely Spaced Parallel Operations (CSPO) Working Group of the Federal Aviation Administration is investigating operational concepts to allow simultaneous paired approaches at runways separated by as little as $700 \mathrm{ft}^{1,2}$ In a simultaneous paired approach, a pair of aircraft flies in close proximity to each other as they approach parallel runways. The aircraft pair must maintain longitudinal separation within a range that avoids wake encounters and, if one of the aircraft blunders, also avoids collision. Wake avoidance defines the rear gate of the longitudinal separation. The lead aircraft generates a wake vortex that can, with the aid of crosswinds, travel laterally onto the path of the trail aircraft. As runway separation decreases (and, therefore, the lateral separation of the parallel approach paths decreases), the wake has less distance to travel to cross the path of the trail vehicle. The lateral total system error (TSE) of each aircraft further shortens this distance. TSE characterizes how far from its intended path each aircraft may deviate. TSE is often modeled as statistical variation and typically is defined using a probability density function (PDF). ${ }^{1,6}$ Therefore, Monte-Carlo simulations are a favored tool for assessing the wake-safe gate. However, definitions of safety used for such operations often require that a wake encounter be a very rare event during normal operation; one catastrophic wake encounter per billion flights 


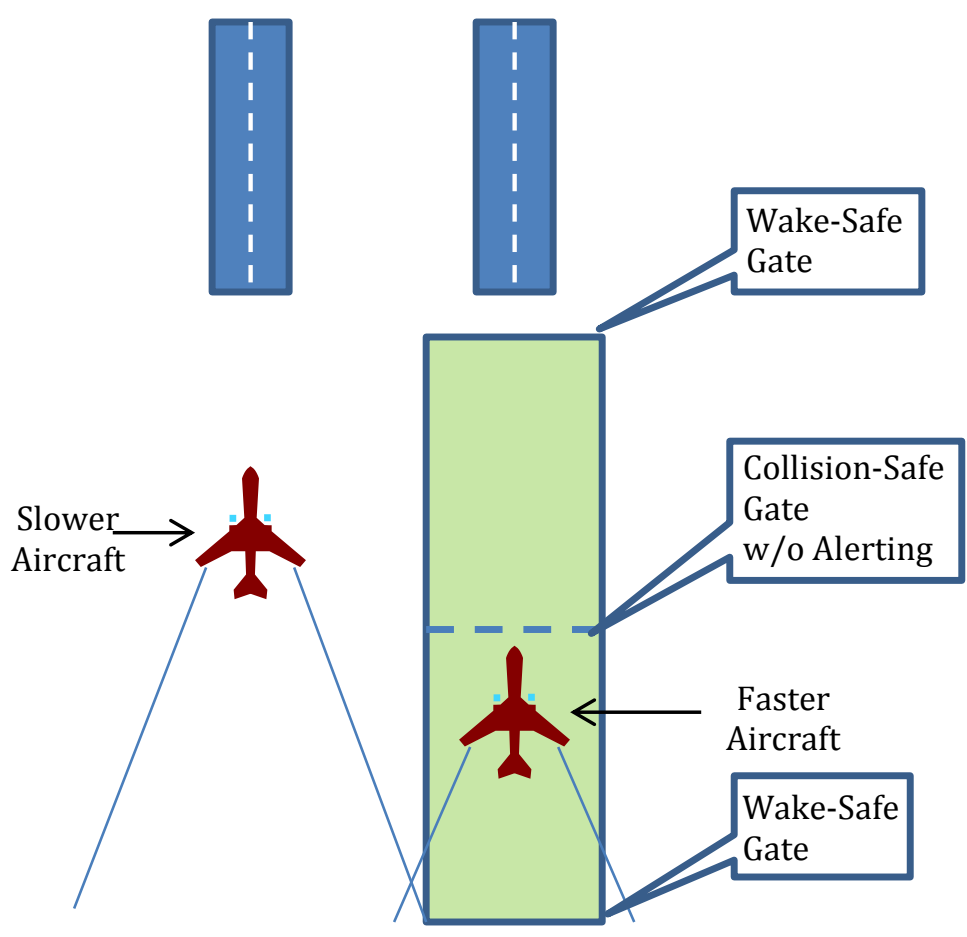

Figure 1: Paired Approach Separation Distances

is typical. ${ }^{6}$ Using a Monte-Carlo simulation to assert the required rarity of a wake encounter with confidence, therefore, requires a massive number of runs. Even for low-fidelity, fast-time simulations on modern computers, such runs do not lend themselves to rapid turn-around during the early stages of investigation when the goal is to eliminate the infeasible regions of the solution space and to perform trades among the independent variables in the operational concept. Statistical analysis using simplified models can be employed more efficiently to narrow the solution space and identify promising trades for more indepth investigation using Monte-Carlo simulations.

Total system error is not the only variation at play in paired approaches. For closely spaced parallel approaches, passive safety is not possible. The operational concepts must rely on active monitoring to ensure conformance with the procedure. For many paired approach concepts, active monitoring relies on ADS-B broadcasts and on-board navigation solutions. Both exhibit uncertainty, which can be statistically modeled. Due to this uncertainty, the alerting threshold is not a hard limit to path deviation. Therefore, the statistical analysis must account for un-alerted deviations beyond the threshold when determining the probability of a wake encounter. This paper presents a method for integrating total system error, surveillance errors ${ }^{\dagger}$, limits on alert rates, and avionics failures into a statistical analysis that identifies the likely feasible runway separations for simultaneous paired approaches.

\section{Approach Geometry}

Candidate geometries for closely spaced parallel approaches are detailed in references 1 through 6 . This paper presents a model to investigate parallel approaches by the aircraft pair. Prior to the start of the procedure, air traffic control vectors a pair of aircraft onto parallel approach paths at a point between the initial approach fix (IAF) and the final approach fix (FAF). The aircraft with the faster final approach speed is placed behind the aircraft with the slower final approach speed. The aircraft also begin with a vertical separation of $1000 \mathrm{ft}$ to allow each aircraft to safely establish their approach path and to allow the fast aircraft to establish longitudinal separation from the slow aircraft. The fast aircraft can start at either the higher altitude or the lower altitude. As the procedure starts, each aircraft flies at a constant altitude

$\dagger$ This is the error between the actual relative position of the paired aircraft, and the relative position observed by the trail aircraft. 


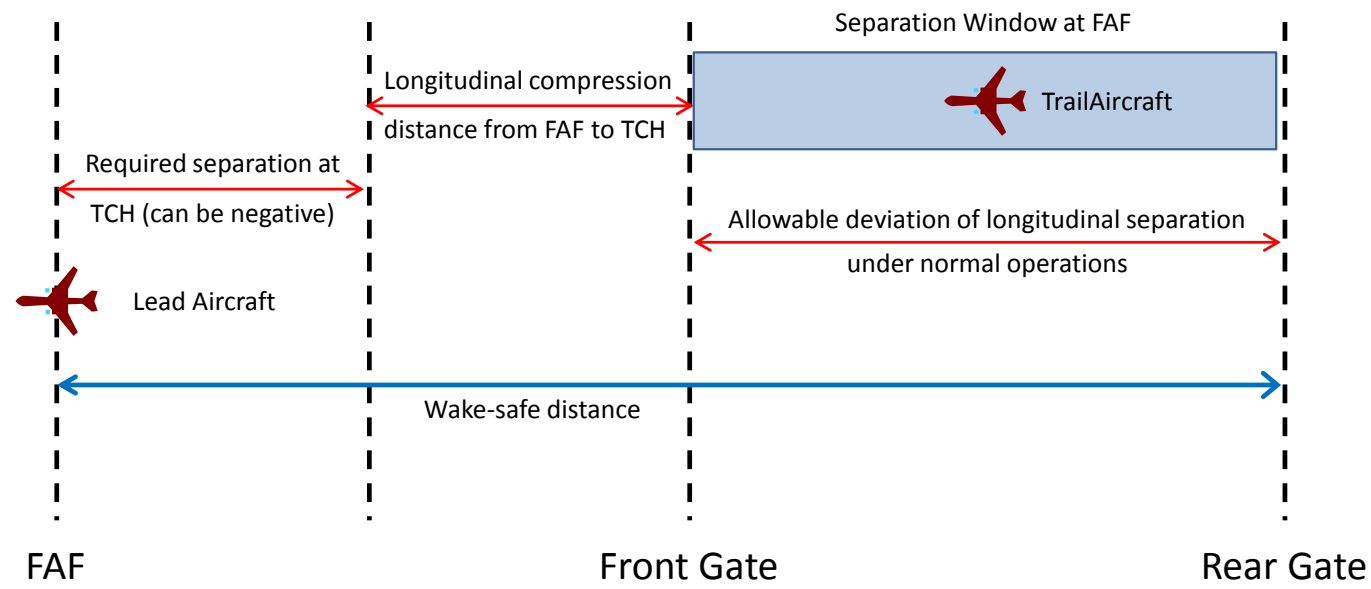

Figure 2. Separation Window at FAF

until encountering the glidepath and then descends along the glidepath. From the start of the procedure to the FAF, the slow aircraft flies an assigned constant speed (nominally $180 \mathrm{KT}$ ) and the fast aircraft maintains speed and position against the slow aircraft. The fast aircraft executes this dependent operation until it reaches its final approach speed (matching speed of the slow aircraft as the slow aircraft decelerates upon reaching the FAF) or, if the fast aircraft reaches the FAF before detecting deceleration of the slow aircraft, the fast aircraft switches to independent operation at the FAF.

Figure 1 depicts the longitudinal geometry of the paired-approach procedure, either with the option of passing ${ }^{1}$ or without the option of passing ${ }^{6}$. The fast aircraft must maintain a longitudinal distance from the slow aircraft in a range defined to avoid two hazards. In the case where passing is not allowed, those hazards are collision and wake. To avoid collision, the fast aircraft must maintain a sufficient distance behind the slow aircraft such that the slow aircraft will not collide with the fast aircraft if the slow aircraft blunders across the fast aircraft's path. The collision-safe gate is defined to be passively safe, i.e. the trail aircraft does not monitor the lateral separation between aircraft. To avoid the wake hazard, the fast aircraft must be close enough to the slow aircraft that the wake of the slow aircraft passes behind the fast aircraft in the presence of adverse crosswind. For the procedure option that allows passing, the two hazards are both wake encounters. The fast aircraft must remain forward of a rear wake-safe gate to avoid encountering the wake of the slow aircraft. The fast aircraft must also remain behind a forward wake-safe gate so that the slow aircraft does not encounter the wake of the fast aircraft. The paired approach with passing relies on collision alerting rather than longitudinal separation to prevent collisions when one or both aircraft blunder. With or without passing, the fast aircraft must maintain its separation between the forward and rear gates from the loss of altitude separation until the slow aircraft crosses the threshold.

The hazard-defined gates define separation windows of considerable length. However, the usable separation window at any given distance along the procedure is often smaller. ${ }^{3,4}$ The cause is a decrease in longitudinal separation that occurs on final approach, after the fast aircraft switches to independent operation. This decrease in separation is a function both of the difference in approach speeds and of the separation limits that must remain at the runway threshold. This decrease in separation forces the front gate of the separation window to retreat further back relative to the slow aircraft as one retraces the fast aircraft's path away from the threshold. Even in the paired approach with passing, this retreat often constrains the fast aircraft to remain in a trail position until late in the approach. Thus, for much of the approach, the paired approach with or without passing has similar geometry that differs only in the location of the front gate of the separation window. For paired approaches with passing, the front gate is simply the distance that prevents the fast aircraft from driving its wake into the trail aircraft before the trail aircraft lands (i.e. it prevents the fast aircraft from flying too far ahead of the slow aircraft before the procedure

* Both collision and wake risks are assumed to be negligible after the lead aircraft crosses the threshold. The assumption on collision risk is reasonable given that ground hazards restrict banking by the lead aircraft and the go-around procedure can be designed to increase lateral separation. However, further wake studies on landing and go around scenarios are needed to validate the assumption of negligible wake risk. 
ends). For the paired approach without passing, the front gate is the distance that prevents the trail aircraft from losing passive safety against collision before the procedure ends. Thus, for both variations of paired approach, the separation window at the final approach fix (FAF) can be defined as show in Fig. 2.

Once the front and rear gate have been estimated, the question remains whether the resulting separation window is long enough to accommodate the deviation in longitudinal separation that can occur under normal operation. Otherwise, the procedure may suffer an ineffectual rate of aborts.

\section{Modeling the Paired Approach with Errors and Uncertainties}

The analytical model presented here uses a target limit on nuisance aborts to define the normal allowable deviation in longitudinal separation. The longitudinal length of the separation window also depends on other variables subject to deviation or uncertainty. These variables include the total system error (TSE) of each aircraft (including its components of flight technical error [FTE] and navigation error $[\mathrm{NE}]$ ), the error in the ADS-B reported position of the slow aircraft, and the deviation in the actual airspeeds of each aircraft. Therefore, given a probability distribution function (PDF) for these variables, one can statistically analyze the length of the separation window given a target probability of successful completion. The feasibility for a given pair of runways becomes a matter of comparing this computed window length against the length remaining from front gate and rear gate estimates. However, the front gate, rear gate, and window length computations are not independent. All three depend to some degree on the TSE of each aircraft. ${ }^{1,4,6}$ Because a statistical model of the wake-safe distance (i.e. the rear gate) can be developed in common for both variations of paired approach, that model is presented here to show how it interacts with the window length. Computation of the front gate is detailed in Ref. 4 and will be presented here as an input parameter.

The examples in this paper present estimates of the front gate, rear gate, and separation window at the final approach fix (FAF), before dependent operation ends and the flexibility of the trail aircraft to adjust its longitudinal separation diminishes. The examples present a paired approach to runways $28 \mathrm{~L}$ and $28 \mathrm{R}$ at San Francisco (KSFO).

\section{A. The Front Gate}

The estimation of the front gate is divided into two lengths as shown in Fig. 2, the estimate of longitudinal separation compression on the final approach and the estimate of the separation limits at the threshold. The model of separation compression is common between the two procedure options, and is detailed in a companion paper ${ }^{7}$ and in Ref. 4 . However, the model of the separation limit is unique to each procedure option and is detailed in Ref. 4 and Ref. 6. Both references rely on a Monte-Carlo simulation to estimate the separation limit at the threshold. For paired approaches with passing, Johnson et al. estimate a separation limit of $-1000 \mathrm{ft}$ at the threshold for runway separations of 750 feet such as at KSFO, ${ }^{4}$ i.e. the fast aircraft can be ahead of the slow aircraft by $1000 \mathrm{ft}$ at the threshold. For paired approaches without passing, Eftekari et al. estimate that the trail aircraft must maintain a collision-safe separation of at least $+1500 \mathrm{ft}$ prior to the FAF and at least $+750 \mathrm{ft}$ on final approach for runways separated by $750 \mathrm{ft}^{6}$

The separation compression is primarily a function of the slow aircraft final approach speed and the final approach speed difference between aircraft. Additionally, Torres-Pomales et al. apply a worst-case bias between actual difference in approach speed and planned difference in approach speed. ${ }^{4}$ The front gate can be tailored to each aircraft pair in real-time or generalized using the worst case aircraft pairing that will be supported by the procedure. Stassen et al. suggests a minimum approach speed of 120 KT with a maximum difference in approach speeds of $20 \mathrm{KT} .{ }^{8}$ For the paired approach with passing, the worst-case front gate is $\sim 3500 \mathrm{ft}(\sim 0.58 \mathrm{NM})$ if the aircraft pair can limit bias in the actual speed difference (i.e. relative speed) to within $\pm 4 \mathrm{KT}$ of planned. For the paired approach without passing, the worst-case front gate is $\sim 5300 \mathrm{ft}(\sim 0.87 \mathrm{NM})$ using the same limits on speed deviation. The worst-case front gate decreases to $\sim 4400 \mathrm{ft}(\sim 0.72 \mathrm{NM})$ for a maximum approach speed difference of $15 \mathrm{KT}$ and to $\sim 3500 \mathrm{ft}(\sim 0.58 \mathrm{NM})$ for a maximum approach speed difference of $10 \mathrm{KT}^{3}$ The examples in this paper will use the worst-case $3500 \mathrm{ft}$ front gate at the FAF for the paired approach with passing.

\section{B. The Wake-Safe Distance}

\section{Modeling Wake Vortex Transport}

This study uses the wake vortex transport model described by Stassen et al. ${ }^{7}$ The model performs a constant velocity transport of the wake vortex from its generation point to the wake vortex encounter zone 


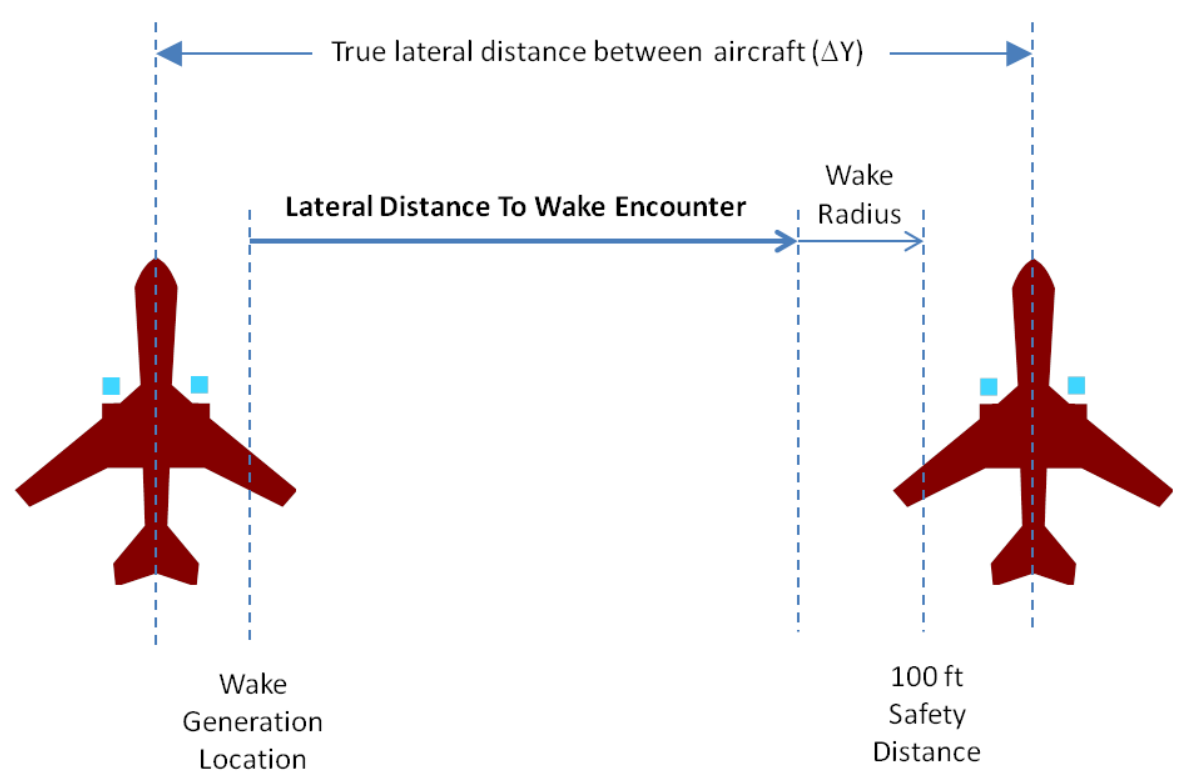

NOT TO SCALE

Figure 3. Wake Transport Model

of the trail aircraft. The transport velocity is the sum of the crosswind velocity and, when below $400 \mathrm{ft}$ AGL, a self-transport velocity of 2 to 3 knots due to ground effects. Fig. 3 depicts the geometry of the lateral distance that the wake vortex can travel before encountering the trailing vehicle. ${ }^{\S}$ The wake encounter zone is a lateral distance from the centerline of the trail aircraft that is the sum of a safe encounter distance of 100 feet $^{* *}$ and the radius of the wake vortex. The distance for the wake to travel is the lateral distance between its generation point and the wake encounter zone.

The following equation expresses the distance to a wake encounter:

$$
\mathrm{D}_{\text {encounter }}=\Delta \mathrm{Y}-\mathrm{D}_{\text {safe }}-\mathrm{R}_{\text {vortex }}-\mathrm{D}_{\text {vortex }}
$$

where,

$\Delta \mathrm{Y} \quad$ is the true lateral distance between aircraft, in feet.

$\mathrm{D}_{\text {safe }} \quad$ is the safe encounter distance from the trail aircraft's centerline, $100 \mathrm{ft}$.

$R_{\text {vortex }}$ is the radius of the vortex which equals $1 / 2 D_{\text {vortex }}$.

$D_{\text {vortex }}$ is the generation location of the vortex from the lead aircraft's centerline. It is equal to ( $\pi$ $D_{\text {wingspan }} / 8$ ) where $D_{\text {wingspan }}$ is the wingspan of the lead aircraft.

The examples will assume the largest lead aircraft is a Boeing 747-400. The Boeing 747-400 has a wingspan of $211 \mathrm{ft} 5 \mathrm{in}$. The distance equation, therefore, reduces to:

$$
D_{\text {encounter }}=\Delta Y-224.5 \mathrm{ft}
$$

The next part of the problem is determining the worst-case (i.e. minimum) $\Delta \mathrm{Y}$ for the paired approach. The worst case $\Delta \mathrm{Y}$ depends on the lateral TSE of each aircraft and is derived in the next section.

${ }^{\S}$ Fig. 3 shows the geometry using the instantaneous true location of each aircraft. Applying a probability density function for the total system error of each aircraft is discussed later.

*** The safe encounter distance is not necessarily the distance that avoids the wake but the distance where a wake encounter does not endanger the aircraft. For a more conservative analysis, one can instead use the half-wingspan of the largest aircraft eligible for the paired approach. 
$\mathrm{D}_{\text {encounter }}$ is then converted to a time-to-encounter $\left(\mathrm{T}_{\text {encounter }}\right)$ by dividing $\mathrm{D}_{\text {encounter }}$ by the wake transport velocity $\left(\mathrm{V}_{\text {transport }}\right) . \mathrm{V}_{\text {transport }}$ is the sum of the maximum allowable crosswind $\left(\mathrm{V}_{\text {crosswind }}\right)$ and, if under $400 \mathrm{ft}$ AGL, the self-transport velocity $\left(\mathrm{V}_{\text {self-transport }}\right)$ :

$$
\mathrm{V}_{\text {transport }}=\mathrm{V}_{\text {crosswind }}+\left\{\begin{array}{cl}
\mathrm{V}_{\text {self-transport }} & \text { if } \mathrm{h}<400 \mathrm{ft} \text { AGL } \\
0 & \text { if } \mathrm{h} \geq 400 \mathrm{ft} \text { AGL }
\end{array}\right.
$$

The examples will assume the maximum allowable $\mathrm{V}_{\text {crosswind }}$ for the paired approach procedure is 10 knots.

Lastly, the longitudinal wake-free gate is the product of the time-to-encounter $\left(\mathrm{T}_{\text {encounter }}\right)$ and the trail aircraft's true ground speed $\left(\mathrm{V}_{\text {trail }}\right)$.

$$
L_{\text {wake-free }}=T_{\text {encounter }} * V_{\text {trail }}=D_{\text {encounter }} * \frac{V_{\text {trail }}}{V_{\text {transport }}}
$$

Equation 4, therefore, models the wake-safe distance as a function of the trail aircraft speed and the TSE of the aircraft (as an input variable to $\mathrm{D}_{\text {encounter }}$ ). The next section details how TSE is applied to determine $\mathrm{D}_{\text {encounter }}$ under a definition of normal operation. Speed is applied using the speed schedule depicted in Fig. 4. The speed schedule consists of three segments: a constant speed segment, a deceleration segment, and a final approach segment. In the constant speed segment, both aircraft fly the same constant speed assigned by air traffic control. Once the lead aircraft reaches the FAF, both aircraft will decelerate to their final approach speed (deceleration segment). Then each aircraft flies their planned final approach speed to the runways threshold (final approach segment). The speed of the trail aircraft is constant in both the constant speed and final approach segments. Therefore, if other model inputs were constant, the wake-safe gate would also be constant in these segments. To limit lateral TSE, the procedure may require a GPS or RNP RNAV leg for this segment because these

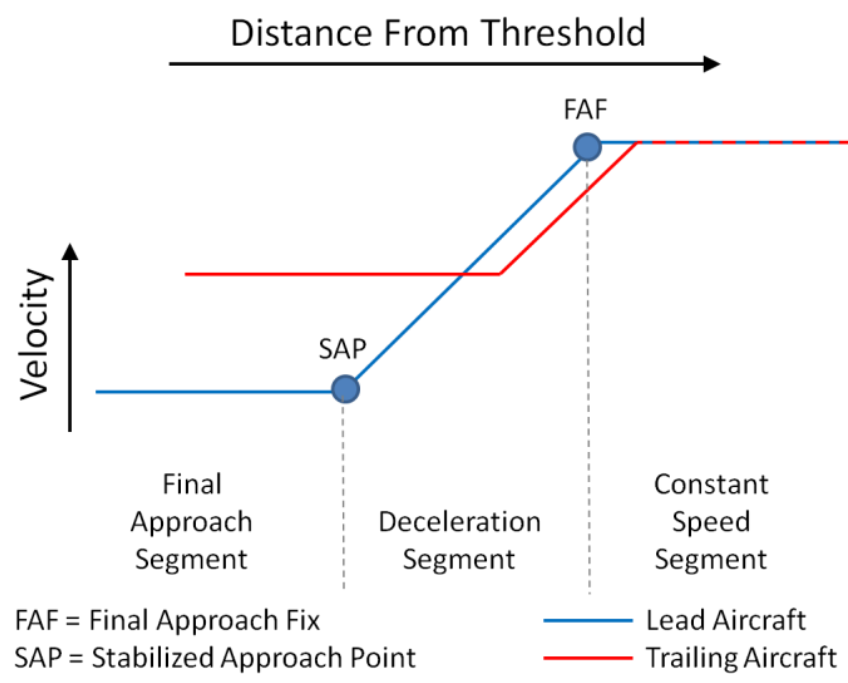

Figure 4 Speed Schedule for Paired Approach legs exhibit standard deviation of TSE that is independent of distance from threshold. Using an ILS navigation mode may be less desirable as the TSE for ILS navigation exhibits a linear increase with distance from the transmitter. ${ }^{8,9}$ However, once past the FAF, the aircraft pair will require a navigation mode that decreases TSE to less than $8 \mathrm{~m}$ (95\% confidence) at the runway threshold, if the paired approach is designed to operate under CAT III conditions. ${ }^{11}$ As TSE decreases, worst-case $\Delta \mathrm{Y}$ increases, which may reduce or reverse the decrease in wake safe distance due to the lower speed in the deceleration and final approach segments. Thus, once the aircraft stabilizes on its final approach speed, the wake-safe distance may remain constant or grow (if TSE is decreasing) until $400 \mathrm{ft}$ AGL when ground effects increase the wake transport speed. Because the constant segment speed is expected to be the same for all aircraft pairs, the wake-safe distance is also the same. Afterwards, the wake safe distance can be customized in real-time to the speed of the trail aircraft, or the wake-safe distance can be generalized by deriving the worst-case from the slowest supported final approach speed. The examples in this paper take place in the constant speed segment as the slow aircraft reaches the FAF.

\section{Closest Lateral Separation under Normal Operations}

Once the speed schedule is established, the closest allowable lateral separation under normal operations $(\Delta \mathrm{Y})$ determines the design-to, wake-free gate for the procedure. The lateral separation between aircraft during the procedure is determined by the actual path each aircraft flies. The total system error (TSE) characterizes the deviation of the actual path from the desired path. TSE is made of three error components: path definition error (PDE), navigation error (NE), and flight technical error (FTE). PDE is 
the difference between the path defined by the on-board avionics and the desired path; it is normally negligible and will be ignored. NE is the error between the aircraft position estimated by the on-board navigation system and the actual position. FTE is the error in the autopilot or crew's ability to fly the path. NE and FTE will both be modeled as a normal distribution for simplicity. The normal distribution is often a good approximation for navigation errors. ${ }^{11-13}$ However, Levy et al. assert that PDFs capable of fatter tails like Johnson curves are a better fit for FTE. ${ }^{14}$ Nevertheless, the data presented by Levy and FTE data for Quantas flights performing RNP approaches presented by Passerini ${ }^{15}$ show that the normal distribution remains a reasonably accurate approximation of FTE and that the normal distribution appears to underestimate actual FTE distributions largely within the range of $\pm 3 \sigma$. The analytical model in this paper will assess deviation bounds in ranges beyond $\pm 4 \sigma$ and, therefore, may overestimate deviation bounds. Since NE and FTE are both modeled as normal distributions, the resulting TSE model will also be a normal distribution. The TSE, NE, and FTE values presented here are 95\% confidence bounds (1.96 $\sigma$ ).

To date, comprehensive studies of FTE for aircraft on approach have examined a mixture of hand-flown and autopilot approaches using the Instrument Landing System (ILS) as the primary navigation aid. Thomas and Timoteo ${ }^{16}$ and Thomas, Timoteo, and Huang ${ }^{17}$ showed that the lateral FTE of ILS approaches increases with distance from threshold. Lateral FTE exceeded $200 \mathrm{ft}$ at distances greater than 8 to $10 \mathrm{NM}$ from the threshold and, on final approach, lateral FTE was less than $40 \mathrm{ft} / \mathrm{NM}$ from threshold. Eckstein also depicted the localizer deviation of ILS approaches starting at $7 \mathrm{NM}$ from threshold and computed the lateral FTE at a point $3 \mathrm{NM}$ from the threshold. ${ }^{18}$ Unlike previous work, Eckstein divided the data between hand-flown and autopilot approaches. The lateral FTE of the autopilot approaches was less than 10 feet and an order of magnitude lower than the hand-flown approaches. Eckstein's study keeps open the possibility that it may be possible for the paired approach procedure to be performed from start to finish using an ILS navigation mode. However, in the absence of a comprehensive analysis of ILS approaches under autopilot and autothrottle alone, this study will proceed under the assumption that the aircraft pair performs an RNP or GPS RNAV approach to the FAF (though each aircraft may transition to an ILS navaid afterwards). Published data on TSE for RNAV approaches is equally scarce. Murphy presents data from Air France showing that the lateral TSE of aircraft on RNP approach was $59 \mathrm{~m}(194 \mathrm{ft})$ at the time they disengaged the autopilot prior to landing. ${ }^{19}$ Passerini provides data on the Quantas fleet of Boeing $737-800$ s that quantified the lateral FTE at 0.02 NM (37 m or $122 \mathrm{ft}$ ) for RNP approaches. ${ }^{15}$ Aircraft performing paired approaches will likely require similar or better performance to that demonstrated by the Quants fleet. Therefore, the examples here use $37 \mathrm{~m}$ as the lateral FTE of both aircraft.

This paper will assume that aircraft rely on GPS or an equivalent navigation system to estimate the position of the aircraft. GPS may either be autonomous or receive corrections from ground or satellite based sources. RTCA DO-242A characterizes the lateral NE of autonomous GPS as $12.5 \mathrm{~m}^{13}$ However,

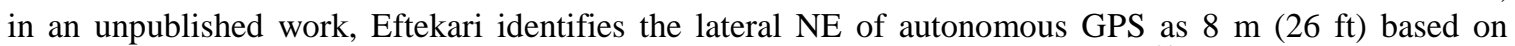
observations from ADS-B flight tests at the FAA Technical Center in July 2007. ${ }^{20}$ RTCA DO-242A also characterizes Wide-Area Augmentation System GPS with a lateral NE of $3.5 \mathrm{~m}(12 \mathrm{ft}){ }^{13}$ The examples here use $3.5 \mathrm{~m}$ as the NE for both aircraft. The resulting TSE for the examples is $37.2 \mathrm{~m}$.

Table 1: Total System Error of Example Aircraft Fleet

\begin{tabular}{|l|l|l|l|l|}
\hline TSE & FTE & NE & Event Duration & Events/Procedure \\
\hline $37.2 \mathrm{~m}$ & $37.0 \mathrm{~m}$ & $3.5 \mathrm{~m}$ & $60 \mathrm{~s}$ & 6 \\
\hline
\end{tabular}

Table 1 summarizes the final TSE, FTE, and NE values. Recognize that these errors are defined for a sample. To ascertain their effect on the maximum deviation over the entire approach path during normal operations, it is necessary to determine how many samples will occur during the course of the procedure. Aircraft do not suddenly jump from one error to the next, the error occurs as a random walk with a period in distance or time. The period of 60 seconds used in the example is in the neighborhood of the period reported by Boeing for its RNP-capable B737-NG aircraft ${ }^{12}$ and the period exhibited in an unpublished model of TSE from Boeing. ${ }^{21}$ For GPS receivers, the period between independent navigation error events is four to eight minutes. ${ }^{22}$ However, because NE is much smaller than FTE, the period of NE events is made equal to FTE events for simplicity. Thus, the period of independent events for TSE, FTE, and NE are each set at 60 seconds. For the example paired approach to San Francisco runways 28L and 28R, the procedure duration is as long as six minutes, dependent on the speed schedule. Therefore, six independent events can occur during the course of the procedure. 
Having established the lateral error characteristics of the aircraft, these characteristics are applied to a definition of normal operation in order to determine the allowable bounds on lateral deviation. Normal operation is defined by an alert rate and alert integrity for each aircraft. Independent monitoring of the lead aircraft by the trail is not practical because ADS-B uncertainties are large relative to the lateral path separation (see section C). Therefore, each aircraft is responsible for monitoring its own lateral deviation. The alert rate is assumed to be $10^{-4}$ alerts per aircraft per procedure without a blunder. Aircraft are capable of monitoring their lateral FTE directly but lateral NE is unknown. Therefore, the actual position of the aircraft can exceed its alerting bounds without an alert. To maintain safety, a containment bound for normal operation is also defined using an alert integrity criterion equivalent to RNP integrity requirements of $99.999 \%$ containment per hour. ${ }^{23}$

First, the alerting bounds are computed. The per-aircraft, per-procedure alert rate ( $\left(\mathrm{ar}_{\text {aircraft }}\right)$ is divided between alerted hardware failure ( $\left(\mathrm{r}_{\text {avionics }}\right)$ and lateral path drift $\left(\mathrm{ar}_{\mathrm{FTE}}\right)$. It is assumed that the hardware will have an alerted failure at a rate of $5 \times 10^{-5} / \mathrm{hr}$. This alerted failure rate is taken from the continuity requirement for RNP of $99.99 \%$ per hour and assumes that half the probability of continuity failure is due to alerted hardware failure. It is also assumed that the hardware alert rate is linear with time and reduces to $\mathrm{ar}_{\text {avionics }}=5 \times 10^{-6} /$ procedure. The remaining alert rate due to lateral FTE is $9.5 \times 10^{-5}$ per procedure. The equivalent alert rate per FTE sample ( $\left(\mathrm{r}_{\mathrm{FTE}}\right.$ ) is given by Eq. (6) where $\mathrm{n}_{\mathrm{FTE}}$ is the number of FTE samples per procedure. This equation is derived from the probability of no alert $\left(1-\mathrm{ar}_{\text {aircraft }}\right)$ being equal to the combined probability of no hardware failure $\left(1-\mathrm{ar}_{\text {avionics }}\right)$ and the probability that the aircraft will not exceed the alerting bound $\left(1-\operatorname{ar}_{\mathrm{FTE}}\right)$ for each of the six FTE samples (Eq. 5). The resulting per FTE sample alert rate is $1.6 \times 10^{-5}$.

$$
\begin{gathered}
\left(1-a r_{\text {aircraft }}\right)=\left(1-a r_{\text {avionics }}\right)\left(1-a r_{F T E}\right)^{n_{F T E}} \\
a r_{F T E}=1-\left(\frac{1-a r_{\text {aircraft }}}{1-a r_{\text {avionics }}}\right)^{1 / n_{F T E}}
\end{gathered}
$$

Using $\operatorname{ar}_{\mathrm{FTE}}$, the FTE bound that triggers an alert is determined from the normal distribution. Though deviations to only one side of the aircraft adversely affect the lateral separation between aircraft, the FTE bound will be computed for alerting on either side of the aircraft path. This is simpler from a procedural perspective. There is no need to identify the aircraft as being on the left or right runway and the alerting remains similar to loss of containment alerts for RNP. The following equation computes the alerting bound $\left(\mathrm{y}_{\text {alert }}\right)$ where $\operatorname{erf}^{-1}$ is the inverse error function:

$$
y_{\text {alert }}=\frac{F T E}{1.96} \sqrt{2} \operatorname{erf}^{-1}\left(1-2 \operatorname{ar}_{F T E}\right)
$$

The resulting $\mathrm{y}_{\text {alert }}$ is $4.16 \sigma_{\mathrm{FTE}}$, beyond the $3 \sigma$ range where a normal distribution exhibits the largest underestimation of the actual FTE distribution.

Next, the alert integrity bound is computed from a target containment probability. The containment probability relates to the safety goal for the procedure. This example uses a containment bound equivalent to the RNP requirement of $99.999 \%$ containment per hour per aircraft, i.e. up aircraft $=10^{-5} / \mathrm{hr}$. The study assumes that integrity loss is divided equally between un-alerted hardware failure (up avionics$_{\text {) }}$ ) and position uncertainty (up $\mathrm{TSE}_{\mathrm{TE}}$ ). Therefore, both contributions have a probability of $5 \times 10^{-6} / \mathrm{hr}$. This probability is further divided into a probability of up avionics $=u_{\text {TSE }}=8.3 \times 10^{-8}$ per FTE sample. The resulting probability of un-alerted containment loss per procedure (inclusive of both aircraft) is $2 \times 10^{-6}$.

The quantity up TSE $_{\text {TS }}$ represents the probability that a) the true position of aircraft $\left(\mathrm{y}_{\text {true }}\right)$ is beyond the integrity bound $\left(y_{\text {integrity }}\right)$ and $b$ ) the aircraft does not generate an alert. This occurs when the navigation error is of a sufficient adverse value to place the estimated position of the aircraft $\left(\mathrm{y}_{\mathrm{FTE}}\right)$ below the alerting bound $y_{\text {alert }}$. Because of the symmetry in the normal distribution of lateral deviation, up ${ }_{\text {TSE }}$ can be formulated as twice the probability, on one side of the path, that $y_{\text {true }}$ will be outside $y_{\text {integrity }}$ when $\left|y_{\text {FTE }}\right|<$ $\mathrm{y}_{\text {alert }}$. Thus, one constructs the combined probability that the navigation error $\left(\mathrm{y}_{\mathrm{NE}}\right)$ is greater than $\mathrm{y}_{\text {integrity }}$ $\mathrm{y}_{\mathrm{FTE}}$ for all $\mathrm{y}_{\mathrm{FTE}}$ in the range $\left[-\mathrm{y}_{\text {alert }}, \mathrm{y}_{\text {alert }}\right]$. This is represented by the dual integral below: 


$$
u p_{T S E}=2 \int_{-y_{\text {alert }}}^{y_{\text {alert }}} \int_{y_{\text {integrity }}-y_{F T E}}^{\infty}\left(\frac{e^{-\frac{y_{F T E}^{2}}{2 \sigma_{F T E}}}}{\sqrt{2 \pi} \sigma_{F T E}}\right)\left(\frac{e^{-\frac{y_{N E}^{2}}{2 \sigma_{N E}}}}{\sqrt{2 \pi} \sigma_{N E}}\right) d y_{N E} d y_{F T E}
$$

where $\sigma_{\mathrm{FTE}}$ and $\sigma_{\mathrm{NE}}$ are the standard deviation of the FTE and NE errors, respectively. Recognizing that the inner integral is equivalent to an integral with the range $\left[-\infty, \mathrm{y}_{\mathrm{FTE}}-\mathrm{y}_{\text {integrity }}\right]$, this integral reduces to:

$$
u p_{T S E}=2 \int_{-y_{\text {alert }}}^{y_{\text {alert }}}\left(\frac{\left(e^{-\frac{y_{F T E}^{2}}{2 \sigma_{F T E}}}\right) \operatorname{erf}\left(\frac{y_{\text {integrity }}-y_{F T E}}{\sqrt{2} \sigma_{N E}}\right)}{2 \sqrt{2 \pi} \sigma_{F T E}}\right) d y_{F T E}
$$

where $\operatorname{erf}()$ is the error function. This outer integral cannot be further reduced and must be resolved numerically. Therefore, the $\mathrm{y}_{\text {integrity }}$ value that corresponds with a up root-finding methods.

The design-to lateral separation bounds for each aircraft are the larger of $y_{\text {alert }}$ and $y_{\text {integrity. }}$. Table 2 summarizes the probabilities used to define normal operation in this section. Table 3 shows the values of $\sigma_{\mathrm{FTE}}, \sigma_{\mathrm{NE}}, \mathrm{y}_{\mathrm{alert}}, \mathrm{y}_{\text {integrity }}$, and the required path separation $(\Delta \mathrm{Y})$, each rounded up to the nearest foot. The required path separation is computed from Eq. (2) where $\Delta \mathrm{Y}=2 \mathrm{x} \max \left(\mathrm{y}_{\text {alert }}, \mathrm{y}_{\text {integrity }}\right)$, i.e. the worst case is when both aircraft deviate toward each other by the design-to separation bounds. The path separation shown here merely depicts the spacing at which the wake vortex does not need to move to encounter the trail aircraft if both aircraft are positioned at their integrity bounds. A feasible runway spacing must be much greater than the value shown. These results show that regardless of front gate constraints, paired approaches for the example aircraft fleet are infeasible for runways separated by less than $767 \mathrm{ft}$ unless the lateral performance of the aircraft fleet improves. The next question is how much longitudinal spacing is needed to enable the procedure, and how much does that longitudinal spacing further expand the feasible runway spacing. To answer this question it is necessary to determine the minimum length of the separation window based on expected longitudinal deviation during normal operation.

Table 2: Summary of Probabilities Defining Normal Lateral Deviation

\begin{tabular}{|c|l|}
\hline Total Alert Rate $\left(\mathrm{ar}_{\text {aircraft }}\right)$ & $10^{-4} /$ aircraft / procedure \\
\hline Hardware Alert Rate $\left(\operatorname{ar}_{\text {avionics }}\right)$ & $5 \times 10^{-6} /$ procedure \\
\hline \multirow{2}{*}{ Position Alert Rate $\left(\mathrm{ar}_{\mathrm{FTE}}\right)$} & $9.5 \times 10^{-5} /$ procedure \\
\cline { 2 - 2 } & $1.583 \times 10^{-5} /$ FTE sample \\
\hline Total Un-alerted Lateral Containment Loss $\left(\mathrm{up}_{\text {procedure }}\right)$ & $2.3 \times 10^{-6} /$ procedure \\
\hline \multirow{2}{*}{ Loss due to un-alerted hardware failure $\left(\right.$ up $\left._{\text {avionics }}\right)$} & $5.0 \times 10^{-7} /$ procedure \\
\cline { 2 - 2 } & $8.3 \times 10^{-8} /$ FTE sample \\
\hline \multirow{2}{*}{ Un-alerted loss due to position uncertainty $\left(\mathrm{up}_{\mathrm{TSE}}\right)$} & $5.0 \times 10^{-7} /$ procedure \\
\cline { 2 - 2 } & $8.3 \times 10^{-8} /$ FTE sample \\
\hline Number of FTE samples per procedure $\left(\mathrm{n}_{\mathrm{FTE}}\right)$ & 6 \\
\hline Duration of FTE sample & 60 seconds \\
\hline
\end{tabular}

Table 3: Example Lateral Deviation Parameters under Normal Operation

\begin{tabular}{|l|l|}
\hline$\sigma_{\mathrm{FTE}}$ & $62 \mathrm{ft}$ \\
\hline$\sigma_{\mathrm{NE}}$ & $5.9 \mathrm{ft}$ \\
\hline$y_{\text {alert }}$ & $258 \mathrm{ft}$ \\
\hline yintegrity & $271 \mathrm{ft}$ \\
\hline path separation & $>>767 \mathrm{ft}$ \\
\hline
\end{tabular}




\section{Minimum Longitudinal Window under Normal Operations}

The minimum longitudinal window during normal operations is defined similarly to the expected lateral deviation during normal operations in the previous section. Normal operation is defined by a desired alert rate and an alert integrity criterion similar to lateral operation. Here, the trail aircraft monitors its position relative to the lead aircraft and attempts to maintain a desired separation. As with lateral monitoring, the desired alert rate is to be no greater than $10^{-4}$ per procedure. Alert integrity due to position uncertainty will also be maintained to the same requirement as RNP integrity.

One difference in the longitudinal monitoring is that the observed longitudinal separation is subject to both the ADS-B OUT error of lead aircraft and the longitudinal TSE of both aircraft. Longitudinal TSE and its components are assumed to equal the lateral TSE, FTE, and NE in this example, but they can have different values in the model. The same assumption is made for the duration of a longitudinal TSE event (60 seconds) and the number of longitudinal TSE events in a procedure (6). The uncertainty in ADS-B reported position has three components: navigation error (NE), latency compensation error, and uncompensated latency error. ADS-B OUT data is assumed to derive from the lead aircraft's navigation solution and, therefore, share the same NE. The two latency errors are combined into an ADS-B latency error (ALE). The combination of NE and ALE is the estimated position uncertainty (EPU) of the ADS-B OUT record. ADS-B EPU is normally reported as a 2-D radius uncertainty and is assumed to conform to a Rayleigh distribution. ${ }^{22}$ Therefore, the standard deviation of uncertainty in longitudinal position $\left(\sigma_{\mathrm{EPU}}\right)$ is EPU/2.447. The FAA ADS-B rule requires a maximum EPU of $92.6 \mathrm{~m}^{24}$ However, to enable paired approaches at runway spacing less than $1000 \mathrm{ft}$, it is assumed that EPU must be limited to $10 \mathrm{~m}$, a Navigation Accuracy Category for Position (NACp) value of $10 .^{4}$

These surveillance errors determine the variation in observed separation to the planned separation. The observed separation is the difference in the observed position of the lead aircraft (via ADS-B OUT) and the observed position of the trail aircraft (via its navigation system). The ADS-B OUT report represents the lead aircraft's observation of its own longitudinal FTE plus the ALE. The trail aircraft uses observation of its own position to monitor its longitudinal FTE. Therefore, the variation in observed longitudinal separation is a combination of both aircraft's FTE and the ALE of the lead aircraft:

$$
\sigma_{\text {obs }}=\sqrt{\left(\sigma_{F T E}\right)_{\text {lead }}^{2}+\left(\sigma_{F T E}\right)_{\text {trail }}^{2}+\left(\sigma_{\text {ALE }}\right)_{\text {lead }}^{2}}
$$

The standard deviation of ALE is computed by subtracting the contribution of NE to EPU:

$$
\sigma_{A L E}=\sqrt{\sigma_{E P U}^{2}-\sigma_{N E}^{2}}
$$

The longitudinal alert uses similar probability assumptions to the lateral alert. The longitudinal alert rate is $10^{-4}$ per procedure with alerted hardware failures occurring with a probability of $5 \times 10^{-6}$ per procedure. ${ }^{\dagger \dagger}$ Therefore, alerts due to separation performance $\left(\mathrm{ar}_{\text {sep }}\right)$ are $9.5 \times 10^{-5}$ per procedure or $1.583 \mathrm{x}$ $10^{-5}$ per FTE sample. During dependent operation (i.e. before the fast aircraft reaches its final approach speed), there are two contributors to deviation in separation performance. The first is the variation in observed separation. The second is the response time $\left(\mathrm{t}_{\text {delay }}\right)$ of the fast aircraft to the slow aircraft. During $t_{\text {delay }}$, the airspeed difference between the two aircraft can vary. Torres-Pomales et al. provide estimates of $t_{\text {delay }}$; the examples in this paper will assume $t_{\text {delay }}$ is 3.5 seconds. ${ }^{3}$ For this study, the longitudinal airspeed difference is assumed to vary with a standard deviation $\left(\sigma_{\Delta \mathrm{V}}\right)$ of $3.4 \mathrm{KT}(5.7 \mathrm{ft} / \mathrm{s}$ or $1.7 \mathrm{~m} / \mathrm{s})$; this number is derived from an assumed $3 \mathrm{KT}$ standard deviation in the horizontal airspeed of each aircraft. The equivalent spatial variation $\left(\sigma_{\Delta x}\right)$ is the product of $t_{\text {delay }}$ and $\sigma_{\Delta v}$. If we assume $t_{\text {delay }}$ is 3.5 seconds then, $\sigma_{\Delta x}$ is approximately $20 \mathrm{ft}$. Therefore the total variation in separation performance $\left(\sigma_{\mathrm{sep}}\right)$ is:

${ }^{\dagger}$ For simplicity, the model defines independent contributions from hardware failures to lateral and longitudinal alerts when it is likely that both alerts rely on the same hardware. When alerts rely on the same hardware, one should apply the contribution from hardware failures to the overall alert rate for the procedure and then divide the remaining alert rate due to drift between lateral and longitudinal components. 


$$
\sigma_{s e p}=\sqrt{\sigma_{o b s}^{2}+\sigma_{\Delta x}^{2}}
$$

The longitudinal alerting bound $\left(\mathrm{x}_{\text {alert }}\right)$ is therefore:

$$
x_{\text {alert }}=\sigma_{\text {sep }} \sqrt{2} e r f^{-1}\left(1-2 a r_{\text {sep }}\right)
$$

The integrity of the longitudinal alert also uses similar probability assumptions to the lateral alert. Unalerted longitudinal containment loss is $10^{-5}$ per hour with the contributions of un-alerted hardware failure

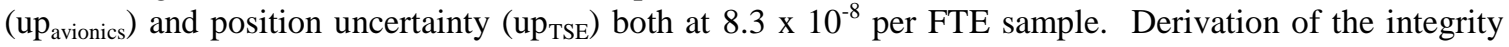
bound $\left(\mathrm{x}_{\text {integrity }}\right)$ is similar to the lateral integrity bound with the following differences. The two probability density functions (PDF) involved are the PDF of separation performance (a function of $\sigma_{\text {sep }}$ ) and the PDF of navigation uncertainty. However, the navigation uncertainty in this case is the combined navigation uncertainty of the two aircraft. Since the navigation uncertainty of the two aircraft is assumed equal, the PDF of the combined navigation uncertainty is a function of $\sqrt{2} \sigma_{\mathrm{NE}}$. The resulting equation for $\mathrm{x}_{\text {integrity }}$ is:

$$
u p_{T S E}=2 \int_{-x_{\text {alert }}}^{x_{\text {alert }}}\left(\frac{\left(e^{-\frac{x_{\text {sep }}^{2}}{2 \sigma_{\text {sep }}}}\right) \operatorname{erf}\left(\frac{x_{\text {integrity }}-x_{F T E}}{2 \sigma_{N E}}\right)}{2 \sqrt{2 \pi} \sigma_{\text {sep }}}\right) d x_{\text {sep }}
$$

The design-to, longitudinal window for the trail aircraft $\left(\mathrm{x}_{\text {window }}\right)$ is double the larger of $\mathrm{y}_{\text {alert }}$ and $\mathrm{y}_{\text {integrity }}$. Table 4 summarizes the probabilities used to define normal operation in this section. Table 5 shows the values of $\sigma_{\mathrm{sep}}, \sigma_{\mathrm{NE}}, \mathrm{x}_{\mathrm{alert}}, \mathrm{x}_{\text {integrity }}$, and $\mathrm{x}_{\mathrm{window}}$, rounded up to the nearest foot for the example aircraft fleet.

Table 4: Summary of Probabilities Defining Normal Longitudinal Deviation

\begin{tabular}{|c|l|}
\hline Total Alert Rate $\left(\operatorname{ar}_{\text {aircraft }}\right)$ & $10^{-4} /$ procedure \\
\hline Hardware Alert Rate $\left(\operatorname{ar}_{\text {avionics }}\right)$ & $5 \times 10^{-6} /$ procedure \\
\hline Position Alert Rate $\left(\operatorname{ar}_{\mathrm{FTE}}\right)$ & $9.5 \times 10^{-5} /$ procedure \\
\cline { 2 - 2 } & $1.583 \times 10^{-5} / \mathrm{FTE} \mathrm{sample}$ \\
\hline Total Un-alerted Lateral Containment Loss $\left(\right.$ up $\left._{\text {procedure }}\right)$ & $1.17 \times 10^{-6} /$ procedure \\
\hline Loss due to un-alerted hardware failure $\left(\right.$ up $\left._{\text {avionics }}\right)$ & $5.0 \times 10^{-7} /$ procedure \\
\cline { 2 - 2 } & $8.3 \times 10^{-8} /$ FTE sample \\
\hline Un-alerted loss due to position uncertainty $\left(\right.$ up $\left._{\mathrm{TSE}}\right)$ & $5.0 \times 10^{-7} /$ procedure \\
\cline { 2 - 2 } & $8.3 \times 10^{-8} / \mathrm{FTE} \mathrm{sample}$ \\
\hline Number of FTE samples per procedure $\left(\mathrm{n}_{\mathrm{FTE}}\right)$ & 6 \\
\hline Duration of FTE sample & 60 seconds \\
\hline
\end{tabular}

Table 5: Example Longitudinal Deviation Parameters under Normal Operation

\begin{tabular}{|l|l|}
\hline$\sigma_{\text {sep }}$ & $97 \mathrm{ft}$ \\
\hline$\sigma_{\mathrm{NE}}$ & $5.9 \mathrm{ft}$ \\
\hline $\mathbf{X}_{\text {alert }}$ & $403 \mathrm{ft}$ \\
\hline $\mathbf{X}_{\text {integrity }}$ & $418 \mathrm{ft}$ \\
\hline$X_{\text {window }}$ & $836 \mathrm{ft}$ \\
\hline
\end{tabular}

\section{Calculating the Wake-Safe Distance}

The required wake-safe distance $\left(\mathrm{L}_{\text {wake-free }}\right)$ is a simple summation of the longitudinal separation window $\left(\mathrm{x}_{\text {window }}\right)$ and the front-gate $\left(\mathrm{x}_{\text {front-gate }}\right)$ :

$$
L_{\text {wake-free }}=x_{\text {front-gate }}+x_{\text {window }}
$$




\section{E. Determining Feasible Runway Separation}

The feasible runway separation can be computed as a function of the required wake-safe distance $\left(\mathrm{L}_{\text {wake- }}\right.$ free $)$ and the lateral integrity $\left(\mathrm{y}_{\text {integrity }}\right)$. First, the required wake transport distance $\left(\mathrm{D}_{\text {encounter }}\right)$ is computed by inverting Equation 4:

$$
D_{\text {encounter }}=L_{\text {wake-free }} \frac{V_{\text {transport }}}{V_{\text {trail }}}
$$

Before the FAF, the worst-case wake transport velocity $\left(\mathrm{V}_{\text {transport }}\right)$ is equal to the maximum allowable adverse crosswind of $10 \mathrm{KT}$. The trail aircraft velocity $\left(\mathrm{V}_{\text {trail }}\right)$ is nominally $180 \mathrm{KT}$ but we will assume that variation in speed performance can decrease it to a minimum of $177 \mathrm{KT}$.

With $\mathrm{D}_{\text {encounter }}$ computed, the minimum runway separation $\left(\Delta \mathrm{Y}_{\text {runway }}\right)$ that is feasible for the procedure is:

$$
\Delta Y_{\text {runway }}=224.5 \mathrm{ft}+D_{\text {encounter }}+2 y_{\text {integrity }}
$$

Table 6 shows the results, rounded up to the nearest foot, for the example aircraft fleet:

\section{Table 6: Feasible Separation Geometry}

\begin{tabular}{|l|r|}
\hline $\mathrm{X}_{\text {front-gate }}$ & $3500 \mathrm{ft}$ \\
\hline $\mathrm{L}_{\text {wake-free }}$ & $4336 \mathrm{ft}$ \\
\hline $\mathrm{D}_{\text {encounter }}$ & $245 \mathrm{ft}$ \\
\hline$\Delta \mathrm{Y}_{\text {runway }}$ & $1011 \mathrm{ft}$ \\
\hline
\end{tabular}

The results show that the example aircraft fleet cannot conduct the paired approach procedure as defined by the selected procedure parameters at runways separated by less than $1011 \mathrm{ft}$. Getting to smaller separations can be achieved by relaxing some of the procedure parameters like rate of alerts, minimum approach speed, or maximum approach speed difference. Likewise, obtaining better data on aircraft technical performance (TSE and speed schedule deviation) or avionics failures may also reduce the minimum feasible runway separations when fed back into this model. However, achieving feasibility on runway separation below $900 \mathrm{ft}$ will likely require technology advances that reduce aircraft TSE by half $(<20 \mathrm{~m}){ }^{3}$

\section{Conclusions}

An analytical model was derived to ascertain the feasible runway separation for a paired approach given procedure parameters that define bounds on normal operation, aircraft performance errors, avionics failure rates, and surveillance errors. To bound normal operation, the model uses a target limit on abort alerts, a target containment probability for position integrity, a maximum allowable crosswind, and a maximum wingspan. Furthermore, the model presented here relies on the algebraic model of Ref. 3 and Ref. 7 to estimate the front gate position. That front-gate model uses additional parameters that bound normal operation, specifically the minimum airspeed of the slow aircraft and maximum approach speed difference of the fast aircraft. Aircraft performance is characterized using normal distributions for flight technical error and navigation error and setting bounds on deviation from speed schedule. Avionics failure rates include fixed probabilities for both alerted and unalerted failures. Surveillance errors are normal distributions of ADS-B estimated position uncertainty that can be taken from the $\mathrm{NAC}_{\mathrm{p}}$ table. Altogether, these parameters cover much of the design and performance space for simultaneous paired approaches. Through its use of probability density functions (PDFs), the analytical model also ensures that results do not represent artificial truncations in the rare probability of extreme performance errors or uncertainties. At the same time, the model is simple enough to script in a mathematical package like Matlab or to implement in a computing language like $\mathrm{C}$. The example results presented here were produced in Matlab, and execution time took seconds. Thus, the model allows investigators to more rapidly narrow the solution space and use more compute intensive Monte-Carlo simulation for validation and refinement. The analytical model cannot replace Monte-Carlo simulations because the analytical model relies on simple kinematic relationships and idealized PDFs. Monte-Carlo simulations are able to provide increasing levels of fidelity to more accurately simulate the performance of real aircraft but at the cost of massive runs to assure that very rare events are represented in the results. 


\section{References}

${ }^{1}$ Johnson, S. C., Abbott, T. S. , Guerreiro, N. M. G, Lohr, G. W., Volk, P., and McKissick, B. T., "Simplified Aircraft-Based Paired Approach: Concept Definition and Initial Analysis," National Aeronautics and Space Administration, NASA/TP-2013-217994, 2013.

${ }^{2}$ Bone, R., O. Olmos, and A. Mundra, "Paired Approach Operational Concept," Proceedings of the 20th Digital Avionics Systems Conference, Daytona Beach, FL, 20001.

${ }^{3}$ Torres-Pomales, W., Madden, M. M., Butler, R. W. and Perry, R. B., "The Simplified Aircraft-Based Paired Approach with the ALAS Alerting Algorithm," NASA/TM-2013-217804, 2013.

${ }^{4}$ Perry, R. B., Madden, M. M., Torres-Pomales, W. and Butler, R. W., "Analysis and Simulation of the Simplified Aircraft-Based Paired Approach Concept With the ALAS Alerting Algorithm in Conjunction With Echelon and Offset Strategies," NASA/TM-2014-218151, 2014, publication pending.

${ }^{5}$ Hammer, J., "Case study of paired approach procedure to closely spaced parallel runways," Air Traffic Control Quarterly, Vol. 8(3), 223-252, 1999.

${ }^{6}$ Eftekari, R. R., Hammer, J. B., Havens, D. A., and Mundra, A.D., "Feasibility analyses for paired approach procedures for closely spaced parallel runways," Integrated Communications, Navigation and Surveillance Conference (ICNS), 2011 , pp. I5-1 - I5-14, 10-12 May 2011.

${ }^{7}$ Madden, M. M., "Kinematic Modeling of Separation Compression for Paired Approaches to CloselySpaced Parallel Runways," AIAA Aviation and Aeronautics Forum and Exposition (AVIATION 2014), Atlanta, June 16-20, 2014.

${ }^{8}$ Stassen, H. P., Eftekari, R., Mundra, A., Domino, D. A., Haltli, B., Koch, M., Lekovish, D., Massimini, V., and Tuomey, D., "Paired Approach Concept of Operations (CONOPS)", MITRE, MITRE Product 130081, Version 1.0, June 28, 2013.

${ }^{9}$ Thomas, J. and Timoteo, D., "Chicago O'Hare Simultaneous ILS Approach Data Collection and Analysis", U. S. Department of Transportation, Atlantic City, NJ, Report DOT/FAA/CT-TN90/11, April 1990.

${ }^{10}$ Thomas, J., Timoteo, D., and Hoang, P., "Los Angeles International Airport Instrument Landing System Approach Data Collection and Reduction Phase 1 Final Report", U. S. Department of Transportation, Atlantic City, NJ, Report DOT/FAA/CT-TN93/12, November 1993.

${ }^{11}$ Kayton, M. and Fried, W. R. Avionics Navigation Systems, John Wiley and Sons, Inc., New York, 1997, ISBN 0-471-54795.

${ }^{12}$ The Boeing Company, "RNP Capability of FMC Equipped 737, Generation 3," Document Number D6-39067-3, Release G, June 12, 2012.

${ }^{13}$ RTCA, Inc, "Minimum Aviation System Performance Standards for Automatic Dependent Surveillance Broadcast (ADS-B)", DO-242A, Washington, D.C., June 25, 2002.

${ }^{14}$ Levy, B.S., Som, P., Greenhaw, R., "Analysis of Flight Technical Error on Straight, Final Approach Segments," Proceedings of the 59th Annual Meeting of The Institute of Navigation and CIGTF 22nd Guidance Test Symposium, Albuquerque, NM, June 2003, pp. 456-467.

${ }^{15}$ Passerini, Alex. "Qantas RNP-AR Data Review, Feb 2011 Update", Qantas Airlines, February 2011, Presentation.

${ }^{16}$ Thomas, J. and Timoteo, D., "Chicago O'Hare Simultaneous ILS Approach Data Collection and Analysis," U. S. Department of Transportation, Atlantic City, NJ, Report DOT/FAA/CT-TN90/11, April 1990.

${ }^{17}$ Thomas, J., Timoteo, D., and Hoang, P., "Los Angeles International Airport Instrument Landing System Approach Data Collection and Reduction Phase 1 Final Report," U. S. Department of Transportation, Atlantic City, NJ, Report DOT/FAA/CT-TN93/12, November 1993.

${ }^{18}$ Eckstein, Adric. "Directed FOQA Studies to Correlate Aircraft Navigation with PBN Procedure", MITRE, MITRE Technical Report 110298, July 2011.

${ }^{19}$ Murphy, Tim. "GNSS Implementation on Commercial Air Transport Airplanes", The 14th Meeting of the APEC GNSS Implementation Team (GIT/14), Seattle, Washington, June 21-24, 2010, Presentation.

${ }^{20}$ Eftekari, R.R., "Terminal Area RNAV/RNP Flight Path Synthesis", MITRE, unpublished, July 31, 2008.

${ }^{21}$ Warren, A. W., "Lateral Control \& Error Modeling for Precision Approach," Boeing Commercial Airplane Group, Revision 3.1, Oct. 27, 1999, unpublished.

${ }^{22}$ Mohleji, S. C., and Wang, G., "Modeling ADS-B Position and Velocity Errors for Airborne Merging and Spacing in Interval Management Application," MITRE, McClean, VA, September 2010, [Online 
Report] URL: http://www.mitre.org/publications/technical-papers/modeling-adsb-position-and-velocityerrors-for-airborne-merging-and-spacing-in-interval-management-application.

${ }^{23}$ RTCA, Inc, "Minimum Operational Performance Standards for Required Navigation Performance for Area Navigation," DO-283A, Washington, D. C., October $28^{\text {th }}, 2003$.

${ }^{24}$ Federal Aviation Administration, "Automatic Dependent Surveillance-Broadcast (ADS-B) Out Performance Requirements To Support Air Traffic Control (ATC) Service; Final Rule," Federal Register (National Archive and Records Administration) 75, no. 103, May 2010, pp. 30160 - 30195. 